\title{
Revistas Científicas em Administração: o papel histórico da Revista de Administração de Empresas (RAE) na construção do campo acadêmico em Administração no Brasil
}

\author{
MARIA JOSÉ TONELLI \\ ${ }^{1}$ Fundação Getulio Vargas / Escola de Administração de EMpresas de SÃo Paulo, Departamento de Administração Geral E
} RECURSOS HUMANOS, SÃo PAULO - SP, BRASIL

\begin{abstract}
Resumo
Este artigo analisa o papel histórico da Revista de Administração de Empresas (RAE) na construção do campo acadêmico em Administração no Brasil, com base nos artigos, nos editoriais e nas resenhas publicadas a partir de sua criação, em 1961. A RAE surgiu como o periódico que introduziu os primeiros modelos de gestão a ser seguidos. Trata-se de uma nova linguagem, não apenas de formas de organizar as vendas ou a produção, de uma nova mentalidade que propõe uma organização social distinta. São características relevantes da RAE, desde sua criação, a divulgação de novas áreas do conhecimento em Administração e a publicação das primeiras pesquisas empíricas sobre a realidade brasileira. Os primeiros números da RAE indicam: i) a presença de autores estrangeiros, vários vinculados à Michigan State University (MSU), instituição que participou da fundação e do desenvolvimento da Escola de Administração de Empresas de São Paulo da Fundação Getulio Vargas (FGV EAESP); ii) os primeiros artigos de áreas temáticas da Administração, em especial, Marketing, Finanças e Comportamento Organizacional; e iii) o contato dos professores brasileiros com uma literatura internacional atualizada, especialmente norte-americana, no campo da Gestão. Ao lado de textos que tratam de práticas administrativas, a RAE também é um espaço para debates político-sociais e artigos reflexivos e críticos.
\end{abstract}

Palavras-chave: Revista de Administração de Empresas. Campo acadêmico. Administração. Mentalidade e gestão.

\section{Scientific Journals in Administration: the historical role of Revista de Administração de Empresas (RAE) in the construction of the academic field of Administration in Brazil}

\section{Abstract}

This article analyzes the historical role of the Revista de Administração de Empresas (RAE) in building the academic field in Administration in Brazil, based on articles, editorials and reviews published since its launch in1961. The RAE appears as the journal that introduces the first management models to be adopted in research, portraying a new language, a new way to organize sales or production, as well as a new mindset that proposes different social organization. Since its launch, relevant features of the journal have been the dissemination of new areas of knowledge in Administration; and the publication of the first empirical research on the Brazilian context. The first issues of RAE indicate: i) the presence of foreign authors, several linked to Michigan State University, an institution that participated in the foundation and development of FGV EAESP; ii) the first articles in thematic areas of Administration, especially Marketing, Finance and Organizational Behavior; and iii) the contact of Brazilian professors with updated international literature, especially North American, in the field of management. Alongside texts dealing with administrative practices, the RAE is also a space for political-social debates and reflective and critical articles.

Keywords: Revista de Administração de Empresas. Academic field. Administration. Mentality and management.

\section{Revistas Científicas sobre Administración: el papel histórico de la Revista de Administração de Empresas (RAE)} en la construcción del campo académico de la Administración en Brasil

\begin{abstract}
Resumen
Este artículo analiza el papel histórico de la Revista de Administração de Empresas (RAE) en la construcción del campo académico de la Administración en Brasil, con base en los artículos, editoriales y reseñas publicados a partir de su lanzamiento en 1961. La RAE surge como la revista que introduce los primeros modelos de gestión que deben seguirse. Se trata de un nuevo lenguaje, no solo de formas de organizar las ventas o la producción, sino de una nueva mentalidad que propone una organización social distinta. Desde su lanzamiento, la divulgación de nuevas áreas de conocimiento en la Administración y la publicación de las primeras investigaciones empíricas sobre la realidad brasileña son características relevantes de la revista. Los primeros números de la RAE indican: i) la presencia de autores extranjeros, varios vinculados a la Michigan State University, institución que participó en la fundación y desarrollo de la FGV EAESP, ii) los primeros artículos de áreas temáticas de la Administración, en especial, marketing, finanzas y comportamiento organizacional y iii) el contacto de los profesores brasileños con una literatura internacional actualizada, especialmente estadounidense, en el campo da gestión. Además de publicar textos sobre prácticas administrativas, RAE es también un espacio para debates político-sociales y artículos reflexivos y críticos.
\end{abstract}

Palabras clave: Revista de Administração de Empresas. Campo académico. Administración. Mentalidad y gestión. 


\section{INTRODUÇÃO}

Logo após a Segunda Guerra Mundial, o Brasil voltava à democracia, com eleições e a promulgação de uma nova Constituição Federal, em setembro de 1946. O país parecia iniciar uma trajetória de modernização e desenvolvimento, com avanços científicos e tecnológicos desenvolvidos nesse período. No cenário internacional, apesar da vitória dos Aliados, com o apoio dos Estados Unidos da América (EUA), a estabilidade mundial estava ameaçada pelo modelo político da antiga União das Repúblicas Socialistas Soviéticas (URSS) e pelo possível confronto entre essas duas grandes potências que se formaram após a guerra (SKIDMORE, 1998; FAUSTO, 2012). Os EUA, preocupados com o papel da URSS, trataram de reorganizar a política mundial de acordo com seus interesses, não só econômicos e políticos, mas também culturais (LEAVITT, 1957; HAINES, 1989). Haines (1989) mostra que várias atividades culturais, além de projetos educacionais e da difusão de livros, com patrocínio de agências americanas, passaram a ser realizadas no Brasil nesse período. Entre 1934 e 1952, a presença da Fundação Rockefeller teve grande influência nas áreas de ensino e pesquisa na Universidade de São Paulo (USP) (MARINHO, 2001). Nesse contexto, a importação de modelos de gestão para o Brasil pode ser considerada a partir dos interesses hegemônicos dos EUA (ALCADIPANI e BERTERO, 2012). Fisher (1984), a propósito da trajetória da Administração Pública no país, revela o mesmo contorno, ou seja, a parceria com órgãos americanos para o desenvolvimento da Administração Pública, inclusive como condição necessária para que fossem feitos investimentos no país. Em 1959, diz a autora, foi assinado com o governo americano um acordo para o Programa de Ensino de Administração Pública e de Empresas (PBA-1), representado pelo Instituto de Assuntos Interamericanos (IAIA) e pela Missão Norte-Americana de Cooperação Técnica (United States Operations Mission - USOM) no Brasil. O PBA-1 incluiu a Fundação Getulio Vargas (FGV), a Universidade Federal da Bahia (UFBA), a Universidade Federal do Rio Grande do Sul (UFRGS), o Ministério de Educação e Cultura (MEC) e o Departamento Administrativo do Serviço Público (DASP), com o intuito de promover a formação de técnicos eficientes que pudessem evitar a corrupção e promover os ideais de racionalidade e liberalismo. Esse período também esteve marcado, como apontam Schwartzmann (2001) e Marinho (2001) por um elemento comum no país: a falta de base para o desenvolvimento de pesquisas científicas de qualidade, o tardio desenvolvimento de instituições para a promoção da ciência, além do conservadorismo das elites no apoio aos campos científicos. Foi nesse contexto que surgiu a RAE - Revista de Administração de Empresas, criada em 1961, em São Paulo, pela FGV.

Este artigo discute o papel da RAE na difusão de repertórios linguísticos de modelos de gestão e na construção do campo científico em Administração. Os periódicos podem ser considerados documentos de época (SHENAV, 1999; SPINK, 1999; PRIOR, 2008) que, ao lado de poderes econômicos e políticos, constituem um poder simbólico que exerce um papel marcante na difusão de ideologias e nas transformações institucionais das sociedades modernas (THOMPSON, 1998; GIDDENS, 2002). Prior (2008) diz que documentos não apenas contêm palavras, informações ou imagens, mas são vozes que influenciam a interação e a organização social (PRIOR, 2008). Nessa mesma linha, Shenav (1999), ao recuperar os artigos publicados em duas revistas da área de Engenharia nos EUA, na virada do século XIX para o XX, reforça a ideia de que o desenvolvimento do modelo americano de gestão deve ser entendido não só como resultado de questões econômicas, mas como produto de forças políticas, culturais e profissionais (SHENAV, 1999).

A RAE teve papel central na difusão de conceitos e práticas da Administração, em um período no qual a circulação de periódicos acadêmicos era praticamente inexistente no país e a mídia popular de negócios também não estava tão difundida. Enquanto a mídia popular de negócios cresceu, lentamente, a partir dos anos 1970 e passou a ter influência na circulação e difusão de valores da racionalidade administrativa, os periódicos acadêmicos em Administração se desenvolveram apenas a partir dos anos 1990 (DONADONE, 2001; WOOD JUNIOR e PAES DE PAULA, 2006, 2008; TONELLI, 2013). No contexto brasileiro da época, a Revista de Administração da Universidade de São Paulo (RAUSP), em 1947, fundadora do campo acadêmico em Administração (TONELLI, 2017) e a RAE, que instituiu as diversas áreas do campo, surgiram como vozes relevantes na disseminação de conhecimento e na construção do campo científico em Administração. Os autores dos artigos publicados na RAE e na RAUSP nesse período foram professores que trabalharam com treinamento e desenvolvimento de executivos, participaram de consultorias e estavam vinculados a instituições políticas. Loureiro (1997), ao discutir o campo das Ciências Econômicas no Brasil, mostra que os economistas migraram das universidades para posições de poder no governo. Na área de Administração, além de artigos, os professores circularam e transitaram "repertórios linguísticos" em consultorias e em treinamento com executivos, trabalharam em práticas de gestão e ajudaram a compor o conceito de um administrador profissional, supostamente eficiente e imparcial na condução dos negócios das empresas. 


\section{A REVISTA DE ADMINISTRAÇÃO DE EMPRESAS - RAE}

A RAE tem sido publicada ininterruptamente desde 1961. O formato gráfico dos primeiros números do periódico é bastante peculiar para uma revista acadêmica, pois trazia anúncios de diversas empresas e de outras publicações da própria FGV (JAYO e MENDES, 2013). O primeiro número traz uma apresentação do então diretor da FGV, Luiz Simões Lopes, do editor da RAE, professor Raimar Richers, e outra do então diretor da Escola de Administração de Empresas de São Paulo da Fundação Getulio Vargas (FGV EAESP), professor Flavio Sampaio, com o título “E.A.E.S.P. - sua origem, suas premissas e seus homens". Tal título não poderia refletir melhor o espírito da época.

\section{Uma nova mentalidade}

Como já apontaram estudos sobre a História da Administração no Brasil (FISHER, 1984; ALCADIPANI e BERTERO, 2012, BARROS, 2013; VALE, 2012), o modelo norte-americano de gestão se faz presente na RAE, com a participação de Vergil D. Reed, professor de Mercadologia da Michigan State University (MSU) e consultor técnico da FGV EAESP, assim como Stanley Bryan, professor de Administração da Produção da MSU, e Leo G. Erickson, professor de Mercadologia e também consultor técnico na FGV EAESP, todos eles autores de artigos no primeiro número da RAE.

O primeiro número da RAE indica: i) a presença de autores estrangeiros, vários vinculados à MSU, instituição que participou da fundação e do desenvolvimento da FGV EAESP; ii) artigos que podem ser identificados com as áreas temáticas da Administração, em especial, Marketing, Finanças e Comportamento Organizacional; e iii) o contato dos professores brasileiros com uma literatura internacional atualizada, especialmente norte-americana, no campo da Gestão.

Os sinais da transposição do modelo americano de gestão para os trópicos também aparecem nos artigos publicados no segundo número da RAE - de setembro a dezembro -, que também apresenta artigos dos professores da MSU que atuam como consultores na FGV EAESP, ao lado de artigos de professores da FGV EAESP. Nesse número, dos 8 artigos publicados, 5 são da área de Comportamento Organizacional, ligados à mudança de mentalidade, tema que aparece, ainda, no artigo "A melhoria da produção no Brasil", de Stanley E. Bryan (1961). O artigo do prof. Sérgio Baptista Zaccarelli (editor da RAUSP a partir de 1977) está voltado a práticas administrativas a ser adotadas nas empresas. Há ênfase na necessidade das empresas adotarem modelos mais profissionais de gestão.

Em 1962, a RAE publicou 3 edições. Os artigos são de autores brasileiros (professores da FGV EAESP) e estrangeiros (da missão da MSU). Alguns aspectos merecem ser ressaltados nesse número:

- Inicialmente, a primeira pesquisa empírica realizada com administradores brasileiros, de autoria de Yolanda F. Balcão e Laerte Leite Cordeiro.

- Em segundo lugar, o artigo de Claude Machline com uma avaliação crítica das empresas, que sofrem dos mesmos males do país (corrupção, inclusive). Os artigos de Balcão, Cordeiro e de Machline, com abordagens críticas e várias referências ao contexto brasileiro, surgem lado a lado com os artigos dos autores norte-americanos e nacionais que buscam trazer modelos de eficiência para a indústria paulista.

- Em terceiro lugar, o artigo de Joseph A. Nordstrom, professor da MSU e também consultor técnico da Faculdade de Ciências Econômicas da Universidade Federal do Rio Grande do Sul (FCE/UFRGS). O artigo de Joseph A. Nordstrom (1962), "Amostragem de trabalho", propõe modelos para a elaboração de padrões de tempo, com o objetivo de evitar tempos improdutivos. A presença de Nordstrom na RAE e a indicação de sua posição como consultor na Universidade Federal do Rio Grande do Sul (UFRGS) mostram, mais uma vez, a presença dos consultores norteamericanos em várias instituições brasileiras (FISHER, 1984; ALCADIPANI e BERTERO, 2012). Além desse artigo, 3 outros - traduções autorizadas - são assinados por professores estrangeiros. A seção de bibliografia também apresenta indicações de livros norte-americanos, incluindo o clássico de Peter Drucker, Landmarks of tomorrow.

Na edição de maio a agosto de 1962 há 3 artigos sobre o empresariado brasileiro (de Raimar Richers, Claude Machline e Luis Carlos Bresser-Pereira). Raimar Richers mostra que há uma ambiguidade dos economistas brasileiros em relação à inflação, no binômio inflação-desenvolvimento. Em consequência, os empresários precisam adaptar-se à inflação. $\mathrm{O}$ artigo é resultado de uma pesquisa realizada com executivos (essa palavra já é utilizada) de 136 empresas brasileiras e financiada pelo Center for International Affairs, da Universidade de Harvard, projeto coordenado pelo também professor da FGV EAESP, Claude Machline. É a segunda pesquisa com dados da realidade brasileira. $\mathrm{O}$ artigo de Claude Machline (coordenador do 
projeto), “O empresário julga o governo", debate as opiniões de um grupo de executivos sobre as políticas do governo para o desenvolvimento da indústria automobilística e conclui por sua aprovação. Bresser-Pereira (1962, p. 90), em “Desenvolvimento econômico e o empresário", discute o conceito de desenvolvimento e a ideia de que "[os] empresários e o Estado são [...] os dois agentes estratégicos do desenvolvimento". São artigos que visam a mobilizar mudanças sociais e econômicas na ação dos empresários e governo. Destaca-se, ainda, o artigo de uma das primeiras acadêmicas brasileiras da área de Marketing, Polia Hamburger, "Produto novo: suas dimensões tecnológicas e de mercado", em que a professora define o que é um produto novo e a necessidade de planejamento cuidadoso, em contraste com a ideia de que "no Brasil ninguém planeja".

O último número publicado em 1962, além de artigos de professores da FGV EAESP, traz artigos dos professores consultores da MSU. Ary Carvalho (1962, p. 13) apresenta um modelo para o treinamento de supervisores, uma vez que estes deveriam repassar aos seus subordinados "os modernos preceitos de administração", para que não haja desperdício dos recursos envolvidos com o treinamento da alta administração. O artigo de Américo Matheus Florentino, professor da Universidade do Estado da Guanabara (UEG) e presidente do Instituto Superior de Contabilidade, trata do "Capital em giro: instrumento de avaliação patrimonial”. Nesse número também há um artigo do superintendente da Companhia Siderúrgica Nacional (CSN), que discute aspectos legais e contábeis e a necessidade da elaboração de normas estatutárias para a administração dos diversos tipos de empresas. Ary Bouzan (1962) discute a questão do subdesenvolvimento do país, no artigo "Mercado interno e desenvolvimento econômico", a partir das propostas de Celso Furtado. Um artigo de Rice (1962) traduzido da Harvard Business Review, por Yolanda Balcão, propõe uma discussão do "Existencialismo para o administrador". Outros artigos (de professores da FGV EAESP e de autores estrangeiros, da missão da MSU na FGV EAESP) são prescritivos e ensinam práticas, como, por exemplo, a determinação de preços de produtos (RICHERS, 1962), a medição da produção (BRYAN, 1961) e potencial de vendas (ERICKSON, 1962). De modo geral, os artigos escritos pelos professores estrangeiros foram traduzidos para o português por professores da FGV EAESP.

Os temas inicialmente abordados pela RAE indicam as principais áreas da Administração: Administração da Produção, Administração Mercadológica, Administração Geral, Administração Financeira, Comportamento Organizacional. A RAE também traz artigos reflexivos e críticos, que questionam a própria área de Administração e o contexto brasileiro, a presença de autores estrangeiros e de traduções em praticamente todos os números da revista e, ainda, artigos sobre formação do administrador e ensino da Administração.

A RAE veicula a influência do pensamento norte-americano em gestão e também mostra estreito contato com a realidade empresarial, com a participação de consultores e diretores de empresas como autores de artigos. Há um claro movimento de levar (e trazer) conhecimento entre as práticas dos negócios e os artigos que estão sendo publicados, que possibilita a circulação de repertórios linguísticos em gestão. É possível identificar artigos claramente direcionados à formação de uma nova "mentalidade" em gestão. A RAE apresenta, ainda, a primeira pesquisa empírica com administradores brasileiros. A presença de autores de outras instituições (USP e UFRGS) mostra que há uma conversação entre professores de várias instituições do Brasil, envolvidos com a construção do campo em Administração.

Quando a RAE foi criada, em 1961, as bases para o desenvolvimento do conhecimento em Administração de Empresas já estavam presentes no país. O periódico reflete essa mentalidade e os artigos que publica, desde sua criação, estão vinculados às áreas temáticas da Administração. A presença de autores norte-americanos, a menção dos autores brasileiros aos estudos conduzidos nos EUA, a tradução de diversos artigos e, em alguns casos, a necessidade de adaptação de termos da área mostram a forte influência dos modelos de gestão norte-americanos na RAE. Apesar de constituir o marco fundacional do campo no Brasil (TONELLI, 2017), a RAUSP não tinha propriamente uma identidade em Administração de Empresas. A RAE surge, então, como o periódico que introduz, de modo claro, os primeiros padrões, os primeiros modelos de gestão a ser seguidos. Trata-se de uma nova linguagem, não apenas da divulgação de novas formas de organizar vendas ou produção, mas de uma mentalidade que propõe uma organização social distinta.

\section{A necessidade de adaptações para o contexto nacional}

A transposição do modelo de gestão norte-americano não ocorreu sem resistências e hibridismos. Questões como o papel do empresariado no Brasil, as necessidades específicas de países subdesenvolvidos e a questão da inflação no desenvolvimento econômico do país, entre outras, estão presentes, com frequência na RAE. Na edição de maio a agosto de 1962 (v. 2, n. 4), por exemplo, há 3 artigos sobre o empresariado brasileiro: Raimar Richers discute "O empresário e a inflação"; Claude Machline 
discute "O empresário julga o governo"; e Bresser-Pereira discute "Desenvolvimento econômico e o empresário". O trabalho de Richers debate se a associação entre desenvolvimento e inflação deve ser considerada uma premissa na economia brasileira. A partir de pesquisa contratada pelo Center for International Affairs, da Universidade de Harvard, Richers, em conjunto com outros professores da FGV EAESP, entrevistou executivos de 136 empresas brasileiras para entender opiniões e atitudes desse grupo em relação à proposta de desenvolvimento econômico conduzida pelo governo. O artigo de Bresser-Pereira discute o relevante papel desempenhado pelos empresários no desenvolvimento econômico do país. O autor utiliza conceitos de Schumpeter para tratar de inovação, também fazendo a distinção entre o papel do empresário e do administrador. Para BresserPereira, não se trata apenas de criar empresas, mas também de desenvolvê-las e, nesse caso, o administrador desempenha papel relevante e inovador no processo.

O artigo de Bouzan (1962) discute a importância do mercado interno no desenvolvimento da economia de países subdesenvolvidos e o caso específico do Brasil, com referência a autores brasileiros como, por exemplo, Celso Furtado, Caio Prado Junior, Antonio Delfim Neto. Bouzan (1962) considera, como Bresser-Pereira, que as empresas e o Estado têm papel importante na organização da estrutura produtiva. Na segunda edição, de 1963, Bouzan (1962) argumenta que a inflação tem maior impacto na classe média e nos trabalhadores rurais do que nos empresários e trabalhadores industriais, já que estes, por caminhos distintos, conseguem repor seus rendimentos. Os empresários porque reajustam seus lucros com rapidez e os operários porque a legislação e as entidades de classe garantem um pouco mais a reposição salarial.

A partir dos anos 1970, pode-se observar na RAE uma série de autores (também intelectuais brasileiros expressivos) que discutem o tema do desenvolvimento: Fernando Henrique Cardoso, José Pastore, Otavio lanni, Mauricio Tratenberg, entre outros. O periódico apresenta espaço alternativo para o debate sobre questões político-sociais no contexto da época do país. Cabe mencionar, por exemplo, os expressivos títulos dos artigos: "Teoria das organizações e as sociedades subdesenvolvidas", de Carlos Osmar Bertero, de 1967; “Considerações críticas sobre uma estratégia política de desenvolvimento", de Laerte Melloni Ziggiatti, de 1969; "As teorias psicossociais do desenvolvimento econômico: comentários críticos", de José Pastore, de 1970; "Desenvolvimento: um desafio social", de Raimar Richers, de 1970; "Dependência, desenvolvimento e ideologia", de Fernando Henrique Cardoso, de 1970; "Ideologia e prática do planejamento durante o Estado Novo", de Otávio lanni, de 1971; "Considerações pouco ortodoxas sobre a função do consumo e suas implicações para os países menos desenvolvidos", de Raimar Richers, de 1971; “Uma versão subdesenvolvida do Admirável Mundo Novo”, de José Paulo Carneiro Vieira, de 1971; "Administração de empresas e desenvolvimento", de Gustavo de Sá e Silva, de 1971; "A teoria geral da administração é uma ideologia” „de Maurício Tratenberg, de 1971; "Política de blocos, segurança e desenvolvimento: uma perspectiva brasileira", de Celso Lafer, de 1972; "Marketing e o bem-estar da sociedade: uma perspectiva para os países subdesenvolvidos", de Paulo Clarindo Goldschmidt, de 1972; "Autogestão: alternativa socialista para o capitalismo", de Antonio Rabaquim e Roberto Antonio Quintaes, de 1972; "Os novos economistas radicais nos Estados Unidos da América”, de Eduardo Matarazzo Suplicy, de 1972; "O controle da transferência de tecnologia para países em desenvolvimento", de Henrique Rattner, de 1973; "Considerações sobre a evolução recente da distribuição de renda no Brasil”, de Rodolfo Hoffmann, de 1973; "Mobilidade social: uma avaliação comparativa", de Luiz Carlos Bresser Pereira, de 1973; "Desigualdades regionais: uma revisão da literatura”, de Luciano Galvão Coutinho, de 1973; “O conceito de capital e a teoria da distribuição de renda”, de Luiz Antonio de Oliveira Lima, de 1974; “Notas sobre o papel das classes médias e do aparelho de Estado nas sociedades em desenvolvimento", de J. A. Guillon-Albuquerque, 1974; Alguns princípios para a modernização tecnológica no Brasil, de José Pastore, de 1974; "Desenvolvimento e emprego: viabilidade de uma tecnologia intermediária", de Henrique Rattner, de 1974; "Ainda sobre a distribuição de renda", de Pedro S. Malan, de 1974; “Um estudo sobre as empresas multinacionais no Brasil”, de Luiz Carlos Bresser Pereira, de 1976.

Se, de um lado, os artigos mostram a difusão dos valores do modelo de gestão norte-americano, por outro, apontam um questionamento da validade da adoção desse modelo na realidade de subdesenvolvimento do país e a necessidade de adaptações. Embora os valores norte-americanos fossem aplaudidos, eles também foram questionados quanto à sua aplicabilidade no contexto brasileiro.

Nesse período, Fernando Prestes Motta também passou a publicar sobre diversos temas em Teoria Organizacional: “O Behaviorismo na Teoria das Organizações", de 1970; "O estruturalismo na teoria das organizações”, de 1970; "A teoria geral dos sistemas na teoria das organizações", de 1971; "Algumas considerações sobre o desenvolvimento organizacional”, de 1971; "Algumas considerações sobre o desenvolvimento organizacional”, de 1972; "A teoria ds Organizações nos Estados Unidos e na União Soviética: introdução a uma análise comparativa", de 1974; “Estrutura e tecnologia: a contribuição britânica", de 1976. É o caso de mencionar, ainda, o artigo de Tratenberg, sobre as questões ideológicas presentes no campo da Administração. 0 autor foi, reconhecidamente, um dos primeiros teóricos críticos em Administração no Brasil (PAES DE PAULA, 2015). 
Embora muitos dos artigos publicados na RAE não tragam qualquer preocupação com a questão da adaptação de conceitos norte-americanos para a realidade local, não se pode negar que muitos artigos de professores da FGV EAESP questionam a transposição imediata de conceitos, que desconsidera características locais. Em síntese, são características relevantes do periódico, desde sua criação, a divulgação de novas áreas de conhecimento em Administração, a publicação de pesquisas empíricas sobre a realidade brasileira. Ao lado de textos que tratam de práticas administrativas, a RAE também constitui espaço de debates político-sociais e artigos reflexivos. Shenav (1999, p. 213), ao pesquisar as revistas de engenharia nos EUA, na virada do século XIX para o XX, mostrou que: "the magazines provided, filtered, and constructed knowledge about their organizational and technical world". Divulgar práticas em Operações ou Finanças e tratar de estilos de liderança ou de modos de conduta para os administradores constituem formas de ver o mundo e agir sobre ele.

\section{CONSIDERAÇÕES FINAIS}

Tal como apontado por Augier, March e Sullivan (2005), no início das escolas de business norte-americanas, os professores eram homens de negócios e não pesquisadores, as escolas estavam voltadas à prática e às pesquisas aplicadas, com dificuldade, inclusive, de ser reconhecidas pela comunidade científica. As primeiras escolas de Administração no Brasil também surgiram ligadas à necessidade de atender à profissionalização requerida pelas empresas nos anos 1950 (BARROS, 2013; VALE, 2012; BERTERO, 2006). A RAE foi o veículo que difundiu os conceitos da racionalidade administrativa no cenário nacional, mostrando pesquisas empíricas e apresentando novas áreas no campo da Administração e práticas de gestão, mas também trouxe artigos reflexivos e críticos, constituindo espaço para debates político-sociais.

O que esse passado nos diz sobre o presente e sobre o futuro da pesquisa em Administração no Brasil? Em primeiro lugar, a dificuldade de fazer pesquisa se faz presente desde os primórdios do campo; segundo, a tradicional oposição entre "teoria" e "prática" precisaria ser analisada em maior profundidade no contexto brasileiro. Apesar do discurso, quase senso comum entre os acadêmicos, de que as pesquisas contemporâneas não contribuem para a prática, não é isso o que se verifica nos primórdios da construção do campo. O processo de construção do campo acadêmico mostra uma característica que talvez se aplique atualmente: os professores são consultores, participam de cursos para desenvolvimento gerencial e, nessas diferentes posições, circulam repertórios linguísticos. A utilização das referências norte-americanas também não é um problema atual está presente desde os primeiros artigos. O que poderia ser interessante pesquisar, na prática, é como os conceitos importados são incorporados à realidade local. Muitas pesquisas brasileiras ainda replicam modelos estrangeiros, sem questionamentos sobre a validade da transposição de conceitos. Temos no presente os mesmos modelos de 50 anos atrás. Há décadas que não somos inventivos nem reproduzimos bem. Ainda hoje é preciso decolonize the mind e produzir pesquisas de qualidade sobre o Brasil e para o Brasil. Podemos ser universais ao falar de nossa própria aldeia. 


\section{REFERÊNCIAS}

ALCADIPANI, R.; BERTERO, C. O. Guerra Fria e ensino do management no Brasil: caso da FGV EAESP. Revista de Administração de Empresas, v. 52, n. 3, p. 284-299, 2012.

AUGIER, M; MARCH, J. G.; SULLIVAN, B. N. Notes on the evolution of a research community: organization studies in Anglophone North America, 1945-2000. Institute for Operations Research and the Management Sciences, v. 16, n. 1, p. 85-95, 2005.

BARROS, A. N. Uma narrativa sobre a história dos cursos de Administração da FACE - UFMG: às margens do mundo e à sombra da FGV? 2013. 186 f. Tese (Doutorado em Administração) - Faculdade de Ciências Econômicas, Universidade Federal de Minas Gerais, Belo Horizonte, 2013.

BERTERO, C. O. Ensino e pesquisa em administração. São Paulo: Thomson Learning, 2006.

BOUZAN, A. Mercado interno e desenvolvimento econômico. Revista de Administração de Empresas, v. 2, n. 5, p. 73-88, 1962.

BRESSER-PEREIRA, L. C. Desenvolvimento econômico e o empresário. Revista de Administração de Empresas, v. 2, n. 4, p. 79-91, 1962.

BRYAN, S. E. A melhoria da produção no Brasil. Revista de Administração de Empresas, v. 1, n. 2, p. 27-55, 1961.

CARVALHO, A. R. O treinamento do administrador para os níveis médio e superior da organização. Revista de Administração de Empresas, v. 2, n. 5, p. 13-21, 1962.

DONADONE, J. C. Os hunos já chegaram: dinâmica organizacional, difusão de conceitos gerenciais e a atuação das consultorias. 2001. 123 f. Tese (Doutorado em Engenharia) - Escola Politécnica, Universidade de São Paulo, São Paulo, 2001.

DRUCKER, P. F. Landmarks of Tomorrow: A Report on the New. New Brunswick: Transaction Publishers, 1996.

ERICKSON, L. G. Índices preliminares do potencial de vendas do Brasil. Revista de Administração de Empresas, v. 2, n. 5, p. 89-104, 1962.

FAUSTO, B. História concisa do Brasil. 5. ed. São Paulo: Edusp, 2012.

FISHER, T. Administração pública como área de conhecimento e ensino: a trajetória brasileira. Revista de Administração de Empresas, v. 24, n. 4, p. 278-288, 1984.

FLORENTINO, A. M. Capital em Giro: Instrumento de Avaliação Patrimonial. Revista de Administração de Empresas, v. 2, n. 5, p. 23-34, 1962.

GIDDENS, A. Modernidade e identidade. Rio de Janeiro: Jorge Zahar, 2002.

GOLDSCHMIDT, P. C. Marketing e o bem-estar da sociedade: uma perspectiva para os países subdesenvolvidos. Revista de Administração de Empresas, v. 12, n. 3, p. 7-15, 1972.

HAINES, G. H. The Americanization of Brazil: a study of U.S. Cold War diplomacy in the third world, 1945-1954. Wilmington: SR Books, 1989.

HAMBURGUER, P. L. Produto Novo: suas Dimensões Tecnológicas e de Mercado. Revista de Administração de Empresas, v. 2, n. 4, p. 111 128, 1962.

JAYO, M.; MENDES, S. R. C. Esse distinto cavalheiro, o administrador brasileiro: cenografia e ethos na publicidade das revistas da FGV-EAESP.
In: ENCONTRO DA ANPAD, 37., 2013, Rio de Janeiro. Anais... Rio de Janeiro, Anpad, 2013.

LEAVITT, H. J. On the export of American management education. The Journal of Business, v. 30, n. 3, p. 153-161, 1957.

LOUREIRO, M. R. Os economistas no governo. Rio de Janeiro: FGV, 1997.

MARINHO, M. G. M. S. C. Norte-americanos no Brasil: uma história da Fundação Rockefeller na Universidade de São Paulo, 1934-1952. Campinas: Autores Associados, 2001.

NORDSTROM, J. A. Amostragem de trabalho. Revista de Administração de Empresas, v. 2, n. 3, p. 29-42, 1962.

PAES DE PAULA, A. P. Estudos organizacionais críticos e pensadores nacionais. Cadernos EBAPE.BR, v. 13, n. 3, p. 410-413, 2015.

PRIOR, L. Repositioning documents in social research. Sociology, v. 42, n. 5, p. 821-836, 2008.

RICE, J. H. Existencialismo para o Administrador. Revista de Administração de Empresas, v. 2, n. 5, p. 125-146, 1962.

RICHERS, R. O Empresário e a Inflação Brasileira. Revista de Administração de Empresas, v. 2, n. 4, p. 31-46, 1962.

SCHWARTZMAN, S. Um espaço para a Ciência: a formação da comunidade científica no Brasil. Brasília: Ministério da Ciência e Tecnologia \& Centro de Estudos Estratégicos, 2001.

SHENAV, Y. Manufacturing rationality: the engineering foundations of the managerial revolution. New York: Oxford University Press, 1999.

SKIDMORE, T. Uma história do Brasil. São Paulo: Paz e Terra, 1998. SPINK, P. K. Análise de documentos de domínio público. In: SPINK, M. J. (Org.). Práticas discursivas e a produção de sentidos no cotidiano. São Paulo: Cortez, 1999. p. 123-152.

THOMPSON, J. B. A mídia e a modernidade: uma teoria social da mídia. Petrópolis: Vozes, 1998.

TONELLI, M. J. Construção social do management: o papel da mídia acadêmica e seus impactos na cultura das organizações. In: MARCHIORI, M. Estudos organizacionais em interface com a cultura. São Caetano do Sul: Difusão, 2013. p. 31-52.

TONELLI, M. J. The foundation of the academic field in business and administration in Brasil: the case of RAUSP. Revista de Administração da USP, v. 52, n. 4, p. 359-362, 2017.

VALE, M. P. E. M. Institucionalização do ensino em administração de empresas na cidade de São Paulo: Um estudo de caso sobre a Faculdade de Economia, Administração e Contabilidade da Universidade de São Paulo (FEA-USP). 2012. 144 f. Dissertação (Mestrado em Administração de Empresas) - Escola de Administração de Empresas de São Paulo, Fundação Getulio Vargas, São Paulo, 2012.

WOOD JUNIOR, T.; PAES DE PAULA, A. P. A mídia especializada e a cultura do management. Organizações \& Sociedade, v. 13, n. 38, p. 91-105, 2006.

WOOD JUNIOR, T.; PAES DE PAULA, A. P. Pop-management literature: popular business press and management culture in Brazil. Canadian Journal of Administrative Sciences, v. 25, n. 3, p. 185-200, 2008.

Maria José Tonelli

Professora titular no Departamento de Administração Geral e Recursos Humanos da Escola de Administração de Empresas de São Paulo da Fundação Getulio Vargas (FGV EAESP). E-mail: maria.jose.tonelli@fgv.br 